CALT-68-2458

hep-th/0310195

\title{
Two-Dimensional Unoriented Strings And Matrix Models
}

\author{
Jaume Gomis and Anton Kapustin \\ California Institute of Technology 452-48, Pasadena, CA 91125
}

\begin{abstract}
We investigate unoriented strings and superstrings in two dimensions and their dual matrix quantum mechanics. Most of the models we study have a tachyon tadpole coming from the $R P^{2}$ worldsheet which needs to be cancelled by a renormalization of the worldsheet theory. We find evidence that the dual matrix models describe the renormalized theory. The singlet sector of the matrix models is integrable and can be formulated in terms of fermions moving in an external potential and interacting via the Calogero-Moser potential. We show that in the double-scaling limit the latter system exhibits particle-hole duality and interpret it in terms of the dual string theory. We also show that oriented string theories in two dimensions can be continuously deformed into unoriented ones by turning on non-local interactions on the worldsheet. We find two unoriented superstring models for which only oriented worldsheets contribute to the S-matrix. A simple explanation for this is found in the dual matrix model.
\end{abstract}

$10 / 2003$ 


\section{Introduction}

The original connection between $D=2$ string theory and matrix quantum mechanics stems from the fact that the Feynman diagrams of the matrix model generate in the double scaling limit the string worldsheet expansion (see [1] [2] [3] [4] for reviews and references). Recently, a more modern perspective on this connection - now called duality - has emerged [5] [6] [7], which relies on the physics of tachyon condensation. In a nutshell, the matrix quantum mechanics is identified with the worldvolume theory on unstable D0-branes in $D=2$ string theory. The connection then follows from the expectation that once the branes disappear via tachyon condensation [8] [9], one is left with the original string theory but in the absence of branes and that in some not fully understood sense the D0-branes can describe the closed string background they were originally immersed in. This new perspective has paved the way for supersymmetric generalizations [10] 111 involving $\hat{c}=1$ string theory 1 , resulting in nonperturbatively consistent string backgrounds. For related work see [12]-[25].

Studying these low dimensional models of string theory may seem a bit academic. Nevertheless, their study in the early 90's resulted in general lessons applicable to more interesting (realistic) string vacua, most notably Shenker's estimates [26] on the contribution of non-perturbative effects. In this paper we consider various two-dimensional models and extract two general lessons from the dual matrix models, which we expect to apply to other string vacua, but that are hard to establish with the present technology. The first general lesson is that models with NS-NS tadpoles can be consistently renormalized to yield calculable, well defined results. Four dimensional, non-supersymmetric models of particle physics constructed from branes generically have NS-NS tadpoles and it is therefore an important issue to address the treatment of tadpole divergences in string theory. For two-dimensional string theory, the matrix model duals give an unambiguous procedure for how to get physically sensible results, providing a hint that the problem with tadpole divergences can be dealt with in higher dimensions. The other general lesson is the high degree of connectivity of string vacua, by which vacua which look very different from the worldsheet point of view can be continuously deformed into each other. In the two-dimensional context considered in this paper, we provide an example of a non-local worldsheet interaction of the type studied in [27] which interpolates between a model of

1 A derivation of this duality using a fishnet diagram expansion is still missing. It would be interesting to see how worldsheet supersymmetry emerges from this point of view. 
oriented strings and a model with an orientifold! Taking these deformations into account is important when discussing the landscape of string theory vacua [28] 229].

In this paper we consider $c=1$ and $\hat{c}=1$ string theory models of unoriented strings, find the corresponding matrix model duals, and test the duality by performing some perturbative computations. Most of the models have a tachyon tadpole on the $R P^{2}$ worldsheet, which result in divergences in the $R P^{2}$ contribution to the S-matrix and in the partition function. Nevertheless, we find evidence that the dual matrix models, which can be reduced to a system of fermions interacting via a Calogero-Moser potential, actually describe string theory propagation in a shifted background, where the string divergences have been cancelled via the Fischler-Susskind [30] [31] mechanism. The matrix model gives an unambiguous regularization of the string divergences and appears to know about the final, finite, stable closed string vacuum of the modell.

We find that the matrix models for unoriented strings can be reached by a particular double-trace deformation of the familiar Hermitian matrix model, which describes oriented strings in two dimensions. Using the dictionary between oriented string theory and the Hermitian matrix model, it is possible to write down a non-local worldsheet deformation [32] [33] 27] responsible for connecting the vacuum with orientable strings to the vacuum with the orientifold.

There is a particular orientifold projection of Type 0B string theory for which we show that the crosscap state vanishes when projected onto physical states. For this model of unoriented strings all contributions from unoriented worldsheets vanish and the S-matrix for the massless tachyon is precisely the same as in oriented Type 0B string theory. This fits nicely with the matrix model description of this orientifold model, which can be shown to reduce to (one-half of) the system of free fermions describing oriented Type 0B strings.

The plan of the paper is as follows. In section 2 we analyze unoriented $c=1$ bosonic string theory and exhibit the need to renormalize the worldsheet theory to cancel divergences arising from a tachyon tadpole on the $R P^{2}$ worldsheet. In section 3 we analyze the matrix model dual and show that it is described by the dynamics of fermions interacting via a Calogero-Moser potential. Using the collective field theory description of this

2 Even in the oriented $c=1$ string model there are divergences due to a tachyon tadpole on the $T^{2}$ worldsheet. Therefore, $T^{2}$ corrections to the S-matrix and higher loop amplitudes are divergent. It is important to renormalize these divergences before comparing with the matrix model amplitudes, which are manifestly finite. It would be interesting to carry this out. 
model we show that the tree amplitudes are the same as in the Hermitian matrix model, as expected from string theory considerations. We find a double trace deformation of the Hermitian matrix model which yields the matrix model for unoriented strings and describe how to write down the non-local worldsheet deformation that interpolates between oriented and unoriented string theory. In section 4 we analyze the thermodynamics of the Calogero-Moser system in an inverted harmonic oscillator potential using the asymptotic Bethe ansatz. We show that there is a particle-hole duality in the model, which implies that the free energies of the dual $S O$ and $S p$ orientifold models are related by flipping the sign of the string coupling constant, $g_{s} \rightarrow-g_{s}$, thus reproducing a well-known property of string theory to all orders in perturbation theory. Section 5 contains a classification of the possible orientifold models of Type 0B string theory together with the computation of the partition function. For one of these models we find that the overlap of the crosscap state with all physical states vanishes, while the other model has divergences due to a tachyon tadpole, as in the bosonic string. In section 6 we construct the matrix model duals for Type 0B unoriented strings and show that the one corresponding to the model with a vanishing crosscap actually reduces to a system of free fermions, thus confirming the string theory expectation that only oriented worldsheets contribute to all orders in perturbation theory. The other matrix model is the same as for the unoriented bosonic string theory, except that now both sides of the inverted harmonic oscillator potential are filled. Section 7 describes possible orientifold projections of Type 0A string theory and the computation of their partition function. Section 8 analyzes the matrix model duals of Type 0A orientifolds; we show that all these models reduce to integrable systems of the Calogero-Moser type.

\section{Unoriented $c=1$ Strings}

The worldsheet description of $D=2$ bosonic string theory is given by a free boson tensored with the Liouville ${ }^{3}$ CFT with $c_{L}=25$. The local dynamics of the Liouville CFT on a worldsheet $\Sigma$ is given by

$$
S=\int_{\Sigma} d^{2} z\left(\frac{1}{2 \pi} \partial \phi \bar{\partial} \phi+\mu e^{2 b \phi}\right),
$$

\footnotetext{
3 As usual, the b,c reparametrization ghosts must also be included.
} 
where $Q=b+\frac{1}{b}$ and $c_{L}=1+6 Q^{2}$. Globally, there is a background "charge" $-Q \chi$ for $\phi$, where $\chi=2-2 g-b-c$ is the Euler characteristic of the worldsheet $\Sigma, b$ is the number of boundaries, and $c$ is the number of crosscaps. The vertex operators corresponding to normalizable states $|p\rangle$ are given by

$$
V_{\alpha}=e^{\alpha \phi} \quad \text { with } \alpha=Q+i p, p \geq 0
$$

When restricting to the zero mode $\phi_{0}$, the wavefunction for $|p\rangle$ - which satisfies the minisuperspace Schrödinger equation - is given by

$$
\psi_{p}\left(\phi_{0}\right)=\frac{2\left(\pi \mu / b^{2}\right)^{-i p / 2 b}}{\Gamma(-i p / b)} K_{i p / b}\left(2 \sqrt{\pi \mu / b^{2}} e^{b \phi_{0}}\right)
$$

in the weak coupling region $\phi_{0} \rightarrow-\infty$ it asymptotes to

$$
\psi_{p}\left(\phi_{0}\right)=e^{i p \phi_{0}}+R^{c l}(p) e^{-i p \phi_{0}}
$$

where $R^{c l}(p)$ is the semiclassical reflection amplitude日. This asymptotics reveals complete reflection off the Liouville potential and gives a rationale for restricting to states with $p \geq 0$.

In $D=2$ string theory asymptotic states are created by the vertex operator

$$
V_{p}=e^{i p X^{0}} V_{\alpha}
$$

and correspond to excitations of a two-dimensional massless scalar field T, known as the massless tachyon.

$D=2$ bosonic string theory is invariant under the action of worldsheet parity $\Omega$, so that one can mode out the theory by the orientifold group $G=\{1, \Omega\}$. The tachyon field $T$ is invariant under the action of $\Omega$ but unoriented worldsheets must now be included (worldsheets $\Sigma$ where the number of crosscaps $c$ does not vanish). At leading order in $g_{s t}$ only the spherical worldsheet contributes, and all scattering amplitudes are the same as in oriented string theoryt.

The leading unoriented Liouville CFT contribution comes from an $R P^{2}$ worldsheet. We first consider the simplest amplitude, the one point function. One can easily compute

\footnotetext{
4 See [34] 35] 36] [37] for details on the exact computation of the reflection amplitude.

5 This well known string theory fact will be deduced in the next section from the dual matrix model viewpoint.
} 
the one point function of $V_{\alpha}$ in the semiclassical regime by constructing in the minisuperspace approximation the wavefunction for the crosscap state $\langle C|$ and evaluating the overlap $\langle C \mid p\rangle$. The crosscap condition [38] [39], when restricted to the zero mode, forces $\langle C|$ to carry zero momentum so that the semiclassical wavefunction is $\psi_{C}\left(\phi_{0}\right)=1$. Therefore, the semiclassical one point function is given by:

$$
\left\langle V_{\alpha}\right\rangle_{R P^{2}}^{c l}=\langle C \mid p\rangle=\int_{-\infty}^{\infty} d \phi_{0} \psi_{C}\left(\phi_{0}\right) \psi_{p}\left(\phi_{0}\right)=\frac{2}{b}\left(\pi \mu / b^{2}\right)^{-i p / 2 b} \Gamma(i p / b) \cosh \left(\frac{\pi p}{2 b}\right) .
$$

The exact computation can be performed by bootstrap methods and gives [40]

$$
\left\langle V_{\alpha}\right\rangle_{R P^{2}}=\left(\pi \mu \gamma\left(b^{2}\right)\right)^{-i p / 2 b} \frac{\Gamma(1+i b p) \Gamma(1+i p / b)}{i p}\left[\cosh \left(\frac{\pi p}{2}\left(b+\frac{1}{b}\right)\right)+\cosh \left(\frac{\pi p}{2}\left(b-\frac{1}{b}\right)\right)\right],
$$

where $\gamma(x)=\frac{\Gamma(x)}{\Gamma(1-x)}$. In the semiclassical regime $(b \ll 1)$ the answer reduces to the minisuperspace computation (2.6).

When considering $D=2$ string theory, one defines the Liouville contribution to amplitudes by taking the following limit

$$
b \rightarrow 1, \mu \rightarrow \infty \quad \text { with } \quad \pi \mu \gamma\left(b^{2}\right)=\mu_{R}
$$

which yields finite results. When $c_{L}=25$, the amplitude (2.7) has poles at imaginary integer values of the momentum which signals the fact that we are dealing with an orientifold plane stretched along the $\phi$ direction, an O1-plane.

In string theory one is interested in the correlators of the on-shell vertex operators $V_{p}$. Energy-momentum conservation forces the one-point function to be evaluated at zero momentum, so that one is computing the zero momentum tachyon tadpole on $R P^{2}$. At first sight, the amplitude (2.7) is divergent in the zero momentum limit due to a pole at $p=0$. Here we interpret this singularity in the CFT computation as a conventional infrared singularity arising from the noncompactness of the target space. The Liouville potential in (2.1) serves as an infrared cutoff in the strong-coupling region and effectively puts the system in a box of length $V_{\phi}=-\frac{1}{2} \log \mu$. The zero momentum limit should be interpreted as inserting the lowest momentum mode in the box, which is given by $p_{\min }=\frac{\pi}{V_{\phi}}$. Therefore, the tachyon tadpole is given by

$$
\lim _{p \rightarrow 0}\left\langle V_{p}\right\rangle_{R P^{2}}=\frac{V_{x^{0}}}{2 \pi} \frac{V_{\phi}}{2 \pi} \cdot C_{R P^{2}}
$$

${ }^{6} C_{R P^{2}}$ is a numerical coefficient which depends on the precise normalization of the crosscap state. 
which agrees with the result obtained by performing a conventional free field theory computation. The fact that the $R P^{2}$ tadpole is captured by free field theory can also be shown by performing the path integral over the Liouville zero mode 41] 42 and noticing that when the vertex operator is at zero momentum, the Liouville interaction can be safely ignored.
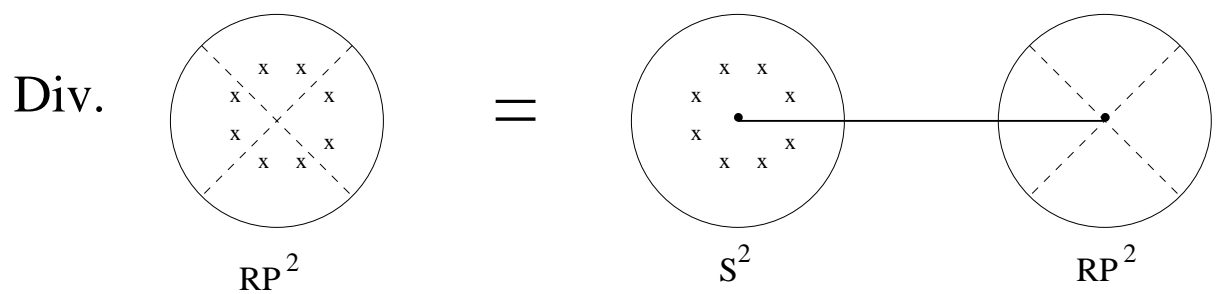

Fig. 1: Origin of the divergence of scattering amplitudes on $R P^{2}$.

A tachyon tadpole on the $R P^{2}$ worldsheet gives rise to divergences in string amplitudes, and some care must be taken in interpreting them. In particular, contributions to the S-matrix ( $n \geq 2$-point functions) arising from the $R P^{2}$ worldsheet are divergent. All these divergences are proportional to the $R P^{2}$ tadpole. The region in the moduli space which yields the divergence is the region where all vertex operators collide. In this region the worldsheet is conformally equivalent to a sphere with all vertex operator inserted connected via a long neck to the $R P^{2}$ worldsheet. The divergence is therefore due to the propagation of the zero momentum tachyon and is proportional to the $R P^{2}$ tachyon tadpole.

Another example of a divergent string amplitude appears in the computation of the free energy of the model, which has a torus and a Klein bottle contribution corresponding to the following partition function:

$$
Z=\operatorname{Tr}\left[\left(\frac{1+\Omega}{2}\right) q^{L_{0}} \bar{q}^{\bar{L}_{0}}\right]=\frac{1}{2} Z_{T^{2}}+\frac{1}{2} Z_{K B}
$$

The torus contribution is well known 43 and yields

$$
Z_{T^{2}}=V_{\phi} \frac{1}{12}\left(\frac{R}{\sqrt{\alpha^{\prime}}}+\frac{\sqrt{\alpha^{\prime}}}{R}\right)
$$

where $V_{\phi}$ is the volume of the Liouville direction, which due to our conventions is $V_{\phi}=$ $-\frac{1}{2} \log \mu$. The first term corresponds to the temperature independent piece of the free 
energy, while the second term is the temperature dependent piece, which can be identified with the free energy of a massless scalar field in two dimensionst.

We now compute the Klein bottle amplitude 8 by performing the path integral 9 . As in the torus computation, the contribution of the massive oscillators cancel the ghost determinant and one is left with a sum over the zero modes of the scalar. We must take into account the action of $\Omega$ on the momentum and winding zero modes

$$
\begin{array}{r}
\Omega|n\rangle=|n\rangle \\
\Omega|w\rangle=|-w\rangle,
\end{array}
$$

so that only momentum modes contribute to the trace. Therefore, one obtains:

$$
Z_{K B}=\frac{V_{\phi}}{2 \pi} \int_{0}^{\infty} \frac{d t}{2 t^{3 / 2}} \sum_{n=-\infty}^{\infty} \exp \left(-\pi t \frac{\alpha^{\prime}}{R^{2}} n^{2}\right)=\frac{V_{\phi}}{2 \pi} \frac{R}{\sqrt{\alpha^{\prime}}} \int_{0}^{\infty} \frac{d t}{2 t^{2}} \sum_{n=-\infty}^{\infty} \exp \left(-\pi \frac{R^{2}}{\alpha^{\prime}} \frac{n^{2}}{t}\right) .
$$

To interpret the amplitude we factorize the Klein bottle into the tree channel $(t=1 / l)$ :

$$
Z_{K B}=\frac{V_{\phi}}{4 \pi} \frac{R}{\sqrt{\alpha^{\prime}}} \int_{0}^{\infty} d l\left(1+2 \sum_{n=1}^{\infty} \exp \left(-\pi \frac{R^{2}}{\alpha^{\prime}} n^{2} l\right)\right)=\frac{V_{\phi}}{4 \pi} \frac{R}{\sqrt{\alpha^{\prime}}}\left(\int_{0}^{\infty} d l+\frac{\pi \alpha^{\prime}}{3 R^{2}}\right) .
$$

There is again a divergence arising from propagation of the zero momentum massless tachyon proportional to the square of the $R P^{2}$ tachyon tadpole. The complete partition function of the model is given by:

$$
Z=V_{\phi} \frac{1}{24}\left(\frac{R}{\sqrt{\alpha^{\prime}}}+2 \frac{\sqrt{\alpha^{\prime}}}{R}\right)+\frac{V_{\phi}}{4 \pi} \frac{R}{\sqrt{\alpha^{\prime}}}\left(\int_{0}^{\infty} d l\right)
$$

Therefore, the temperature independent piece of the free energy is divergent while the temperature dependent piece is precisely that of a massless two dimensional scalar field, as expected from space-time considerations.

7 The free energy is given by $\frac{F}{T}=-Z$, where $T=\frac{\sqrt{\alpha^{\prime}}}{2 \pi R}$. The temperature dependent piece of the free energy of a massless scalar field in two dimensions, which is regularization independent, is given by $F(T)-F(0)=-\frac{\pi}{6} V_{\phi} T^{2}$.

8 See also [44] for the computation of the zero temperature Klein bottle in the $D=2$ bosonic string .

9 One can also compute the Klein bottle by evaluating the crosscap overlap $\int d l\left\langle C\left|e^{-l H_{c}}\right| C\right\rangle$ using (2.7). The naive computation is divergent, but we interpret the divergence as a harmless infrared (volume) divergence just as in (2.9) (the Klein bottle is also proportional to $1 / p_{\text {min }}$ ), and this approach yields precisely the same result as the path integral. 
All the divergences above can be cancelled via the Fischler-Susskind mechanism [30] [31]. This is achieved by adding to the worldsheet Lagrangian a counterterm corresponding to the zero momentum vertex operator - which is precisely the Liouville interaction in (2.1) - with coefficient proportional to the tadpole amplitude in $R P^{2}$. More concretely, we have to renormalize the theory by adding the worldsheet interaction

$$
\delta S_{w s}=\log \Lambda \cdot C_{R P^{2}} \cdot g_{s} \int d^{2} z e^{2 \phi},
$$

where $\Lambda$ is a UV cutoff on the worldsheet. The divergence in the $n$-point function on $R P^{2}$ is now cancelled by the $n$-point function on the sphere with one insertion of (2.16), denoted by $\otimes$, while the Klein bottle divergence is cancelled by the one-point function of (2.16) on the $R P^{2}$ worldsheet. In this way one can construct finite string amplitudes in perturbation theory.

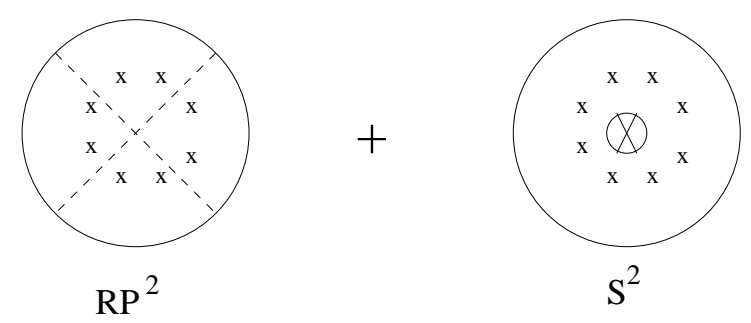

Fig. 2: Cancelling divergence with a counterterm.

\section{Unoriented $c=1$ Matrix Model}

\subsection{Derivation of the matrix model}

The dual matrix model is conjectured to be given by the worldvolume action of $N$ unstable D0-branes in the unoriented model. The basic idea is that once the tachyon mode on the D0-branes condenses, one is left with the original closed string background, and therefore the D0-branes should capture the physics around the tachyon vacuum.

From the work of ZZ [45], it is known that the degrees of freedom of a D0-brane are described by a Hermitian tachyon field $M$ and a non-dynamical gauge field $A$. In order to determine the worldvolume theory on a collection of $N$ D0-branes in the orientifold model, one must demand invariance of the open string vertex operators under the action 
of $\Omega$. The result of the projection depends on the type of orientifold projection under study (depending on the sign of the crosscap state $|C\rangle$ ), and is given by

$$
\begin{aligned}
& O 1^{-}: M^{t}=M \quad A^{t}=-A \\
& O 1^{+}: J M^{t} J=-M \quad J A^{t} J=A,
\end{aligned}
$$

where $J$ is the $S p(N)$ invariant tensor. In the first model $M$ is real and symmetric and $A$ belongs to the $S O(N)$ Lie algebra, while in the second model $J \cdot M$ is antisymmetric and $A$ belongs to the $S p(N)$ Lie algebra.

Thus the effective action of a collection of $N$ D0-branes in this orientifold model is given by the following quantum mechanics model

$$
S=\int d t \operatorname{Tr}\left(\frac{1}{2}\left(D_{t} M\right)^{2}+U(M)\right)
$$

where $D_{t} M=\partial_{t}+i[A, M], U(x)$ is the tachyon potential, and the Hermitian matrices $M(t)$ and $A(t)$ satisfy the constraints (3.1).

\subsection{Reduction to the eigenvalues}

To analyze the dynamics of the system it is convenient to fix a gauge. In all three cases $(U(N), S O(N)$ and $S p(N))$ we may gauge-fix by requiring $M$ to be diagonal [46] [47]. In the $S p(N)$ case each eigenvalue occurs twice. The Faddev-Popov determinant is simply the Jacobian $J$ for the change of coordinates from $M_{i j}$ to the eigenvalues $\lambda_{i}$ and the "angular" variables $U_{j}^{i}$, where the matrix $U_{j}^{i}$ is unitary, orthogonal, or symplectic-unitary. If we denote by $\Delta$ the Vandermonde determinant of the eigenvalues $\lambda_{i}$, given by $\Delta(\lambda)=$ $\prod_{i<j}\left(\lambda_{i}-\lambda_{j}\right)$, the Jacobian is given by:

$$
\begin{array}{cc}
U(N): J=\Delta^{2}(\lambda) & \text { oriented } c=1 \\
S O(N): J=\Delta(\lambda) & \text { unoriented } c=1 \text { with } O 1^{-} \\
S p(N): J=\Delta^{4}(\lambda) & \text { unoriented } c=1 \text { with } O 1^{+} .
\end{array}
$$

The equation of motion for $A$ imposes the Gauss' law constraint and restricts physical states to belong to the singlet sector, where the Hamiltonian simplifies considerably. The Hamiltonian in the singlet sector is given by:

$$
H=-\frac{1}{2 J} \sum_{i} \frac{d}{d \lambda_{i}} J \frac{d}{d \lambda_{i}}+\sum_{i} U\left(\lambda_{i}\right) .
$$


It is convenient to eliminate terms in the Hamiltonian with one derivative by writing $\Psi(\lambda)=\operatorname{sign}(\Delta)|\Delta|^{-\alpha / 2} f(\lambda)$, where $\alpha=2,1,4$ respectively for the cases in (3.3). Then $f(\lambda)$ is an eigenfunction of the following Hamiltonian:

$$
\tilde{H}=-\frac{1}{2} \sum_{i} \frac{d^{2}}{d \lambda_{i}^{2}}+\sum_{i} U\left(\lambda_{i}\right)+\frac{\alpha}{2}\left(\frac{\alpha}{2}-1\right) \sum_{i<j} \frac{1}{\left(\lambda_{i}-\lambda_{j}\right)^{2}} .
$$

Since $\Psi(\lambda)$ is symmetric under the exchange any two eigenvalues, $f(\lambda)$ is antisymmetric. Thus we end up with a system of nonrelativistic fermions in an external potential interacting via the $1 / x^{2}$ potential. This is known as the Calogero-Moser model. For the Hermitian matrix model $(\alpha=2)$, the coefficient multiplying the interaction potential vanishes, and we recover the standard result that the dynamics of the eigenvalues is described by free fermions. We will focus on the values $\alpha=1,4$, which correspond to the quantum mechanics of real symmetric and "quaternionic-Hermitian" matrices (3.1).

We are interested in studying the dynamics of (3.5) in the double scaling limit. In this limit, the only relevant feature of the potential $U(x)$ is the coefficient of the quadratic maximum, which given its interpretation as the tachyon potential, encodes the mass of the open string tachyon [5] [6], so that $U(x)=-\frac{1}{2} x^{2}$. It is well-known that for many purposes, particles in the Calogero-Moser model can be thought of as free, but satisfying a generalized exclusion statistics (see [48] for a review). This means that the number of particles per level cannot be larger than $\alpha / 2$. We will discuss this in more detail in Section 4; for now it is sufficient to say that generalized exclusion statistics implies the existence of a quasi-Fermi surface, much like in the theory of free fermions. Oscillations of the quasiFermi surface can be described by a collective field [49], which is defined as the eigenvalue density:

$$
\phi(x, t)=\sum_{i=1}^{N} \delta\left(x-\lambda_{i}(t)\right)
$$

One also introduces the canonically conjugate momentum $\Pi(x, t)$, which satisfies $[\phi(x, t), \Pi(y, t)]=i \delta(x-y)$. The Hamiltonian for $\phi(x, t)$ and its conjugate momentum $\Pi(x, t)$ is given by 50

$$
H=\int d x\left(\phi \Pi_{, x}^{2}+\frac{\pi^{2} \alpha^{2}}{12} \phi^{3}+\frac{\alpha}{2}\left(\frac{\alpha}{2}-1\right) \phi_{H} \phi_{, x}+\frac{\left(\frac{\alpha}{2}-1\right)^{2}}{4} \frac{\phi_{, x}^{2}}{\phi}+U(x) \phi\right),
$$

where $\phi_{H}$ is the Hilbert transform of $\phi$ :

$$
\phi_{H}(x)=\int d y \phi(y) \frac{P}{x-y},
$$


and $P$ is the principal part. Furthermore, the field $\phi$ is constrained to satisfy:

$$
\int_{-\infty}^{\infty} d x \phi(x)=N
$$

In order to determine the leading large $\mathrm{N}$ behavior, it is useful to define

$$
\phi=\sqrt{N} \hat{\phi}, \quad \Pi=N \hat{\Pi}, \quad x=\sqrt{N} \hat{x}
$$

so that $\int d \hat{x} \hat{\phi}(\hat{x})=1$ and the effective Planck constant is $1 / N^{2}$. Simple scaling shows that the third and fourth terms in (3.7) are subleading in the large $N$ expansion, and that the remaining terms are precisely those of the collective field theory for the Hermitian matrix model (see e.g. [3]), and are proportional to $N^{2}$, as is typical in the tree level approximation. At leading order in $1 / N$ expansion the only difference between the case $\alpha=1$ and the general case is the $\alpha$-dependence of the $\phi^{3}$ term. It can be absorbed into an additional rescaling of $\phi, \Pi$ and $x$. This shows that unoriented string amplitudes at tree level are the same as in the oriented model, as expected from string theory considerations.

\subsection{Connecting oriented and unoriented strings}

One can view the Calogero-Moser system in an inverted harmonic potential as a perturbation of the usual free-fermion system by an interaction term:

$$
H_{\text {int }} \sim \int d x d y \frac{\hat{\rho}(x) \hat{\rho}(y)}{|x-y|^{2}} .
$$

where $\hat{\rho}(x)=\psi^{\dagger}(x) \psi(x)$ is the fermion-density operator. Because of a short-distance singularity in the potential, a regularization is required when doing perturbation theory with this Hamiltonian.

Since free fermions in an inverted harmonic potential are equivalent to the oriented $c=1$ bosonic string, we can regard the Calogero-Moser system in an inverted harmonic potential as a deformation of the latter. In other words, the unoriented $c=1$ string is a deformation of the oriented $c=1$ string!

Let us determine the infinitesimal form of this deformation. It is convenient to switch to momentum space in the $x$-direction. In the language of matrix quantum mechanics, the fermion-density operator $\tilde{\rho}(q, t)$ is:

$$
\tilde{\rho}(q, t)=\operatorname{Tr} e^{i q M(t)}=W_{i q}(t) .
$$


This is known as a macroscopic loop operator (with imaginary length $i q$ ). Thus turning on an interaction potential $V(q)$ between the fermions is equivalent to adding a double-trace operator to the matrix model Hamiltonian:

$$
H_{i n t}=\int \frac{d q}{4 \pi} W_{i q}(t) W_{-i q}(t) V(q)
$$

In the Calogero-Moser case $V(q) \sim|q|$.

Double-trace deformations of the Hermitian matrix model lead to non-local interactions on the worldsheet [32] 33]. Such deformations have recently been studied in the context of AdS/CFT correspondence [27]. To find the explicit form of the non-local interaction, one has to expand the macroscopic loop operator as a linear combination of the tachyon vertex operators as in [51]. Schematically, this gives the following deformation of the action:

$$
\delta S=\int d p V_{p} V_{-p} K(p)
$$

where $K(p)$ is some complicated function whose explicit form is not very illuminating and $V_{p}$ is the tachyon vertex operator. The conclusion is that one can deform the oriented $c=1$ string into the unoriented one by turning on a nonlocal interaction in the worldsheet theory.

\section{Thermodynamics of the Calogero-Moser system in an inverted harmonic potential}

\subsection{Asymptotic Bethe ansatz: a brief review}

We now turn to the thermodynamic properties of the quantum Calogero-Moser model with the goal of comparing with the computations in section 2 . The key feature of the Hamiltonian (3.5) is the existence of $N$ independent integrals of motion whose pairwise commutators vanish [52] [53]. This means that the system is completely integrable and enables one to compute its thermodynamic properties exactly. The usual way of doing this is through asymptotic Bethe ansatz.

Let us recall how this method works in the case when there is no external potential. Complete integrability implies that the many-body S-matrix is elastic and factorizes into a product of two-body S-matrices describing pairwise collisions. Imagine putting the system in a large box $0<x<L$ and let us take the coordinates of all particles but the $i^{\text {th }}$ one to 
be far from the boundaries of the box. Then the wavefunction $f\left(x_{1}, \ldots, x_{N}\right)$, regarded as a function of $x^{i}$, is given by plane-waves near the left boundary of the box:

$$
f\left(x_{i}\right) \sim \sin \left(p_{i} x_{i}\right), \quad x_{i} \simeq 0 .
$$

Then near the right boundary of the box we have:

$$
f\left(x_{i}\right) \sim \exp \left(i p_{i} x_{i}+i \phi_{i}^{+}\right)-\exp \left(-i p_{i} x_{i}-i \phi_{i}^{-}\right)
$$

Here $\phi_{i}^{+}$is the total phase shift resulting from the $i^{\text {th }}$ particle moving to the right with momentum $p_{i}$ and colliding successively with the other $N-1$ particles, while $\phi_{i}^{-}$is the total phase shift resulting from the $i^{\text {th }}$ particle moving to the left with momentum $-p_{i}$ and colliding again with the same particles. In other words

$$
\phi_{i}^{+}=\sum_{j \neq i} \phi\left(p_{i}-p_{j}\right), \quad \phi_{i}^{-}=\sum_{j \neq i} \phi\left(p_{i}+p_{j}\right),
$$

where $\phi(p)$ is the 2-body phase-shift. Requiring the vanishing of the wavefunction on the boundary $x=L$, we get the Bethe equations:

$$
2 p_{i} L+\sum_{i \neq j} \phi\left(p_{i}-p_{j}\right)+\sum_{i \neq j} \phi\left(p_{i}+p_{j}\right)=2 \pi n_{i} \quad n_{i} \in \mathbf{Z} .
$$

This system of equations determines the allowed values of $p_{i}$. Note that $p$ and $-p$ are physically indistinguishable, so we may assume that all $p_{i}$ are positive. In the thermodynamic limit $N \rightarrow \infty$ the allowed values of momenta are very closely spaced, and we may introduce the level-density:

$$
\rho\left(p_{i}\right)=\frac{\Delta n_{i}}{\Delta p_{i}}
$$

For free particles, this density is equal to $L / \pi$. We may formally continue $\rho(p)$ to negative values of $p$ as an even function. The Bethe equations imply a linear integral equation for $\rho(p)$ which takes account of the interactions:

$$
2 \pi \rho(p)=2 L+\int_{-\infty}^{+\infty} \phi^{\prime}(p-q) \rho(q) d q
$$

In the derivation it was assumed that all successive energy levels are occupied by particles, so that the energy

$$
E=\sum_{i} p_{i}^{2}=\int_{0}^{\infty} p^{2} \rho(p) d p
$$


is minimized. For non-zero temperature we are supposed to minimize the free energy instead. Then we must allow holes (unfilled levels) in the particle distribution. Introducing the density of holes $\rho_{h}(p)$, we get the following equation:

$$
2 \pi\left(\rho(p)+\rho_{h}(p)\right)=2 L+\int_{-\infty}^{+\infty} \phi^{\prime}(p-q) \rho(q) d q .
$$

This must be supplemented by a relation between $\rho$ and $\rho_{h}$ obtained by minimizing the free energy. The latter is given as a functional of $\rho$ and $\rho_{h}$ :

$F=E-\mu N-T S=\int_{0}^{\infty}\left\{\left(p^{2}-\mu\right) \rho(p)-T\left[\left(\rho+\rho_{h}\right) \log \left(\rho+\rho_{h}\right)-\rho \log \rho-\rho_{h} \log \rho_{h}\right]\right\} d p$.

In general, this leads to a non-linear integral equation for $\rho$. Once its solution is found, one can find the free energy by substituting $\rho$ and $\rho_{h}$ into (4.9).

If instead of a simple impenetrable box we have a boundary potential at one end, and if this potential preserves the integrability of the system, then the Bethe equations are modified as follows:

$$
2 p_{i} L+\phi_{b}\left(p_{i}\right)+\sum_{i \neq j} \phi\left(p_{i}-p_{j}\right)+\sum_{i \neq j} \phi\left(p_{i}+p_{j}\right)=2 \pi n_{i}, \quad n_{i} \in \mathbf{Z}
$$

Here $\phi_{b}(p)$ is the phase-shift describing reflection off the boundary potential. In the thermodynamic limit we can replace this equation by a linear integral equation for $\rho$ and $\rho_{h}:$

$$
2 \pi\left(\rho(p)+\rho_{h}(p)\right)=2 L+\phi_{b}^{\prime}(p)+\int_{-\infty}^{+\infty} \phi^{\prime}(p-q) \rho(q) d q
$$

4.2. Asymptotic Bethe ansatz for the Calogero-Moser system in an inverted harmonic potential

In the case of interest to us (Eq. (3.5)) the role of the boundary potential is played by the inverted harmonic oscillator potential $U(x)=-\frac{1}{2} x^{2}$. This entails certain modifications in the arguments. Firstly, the inverted harmonic potential allows for tunneling to the other side of the potential; however, if we are interested in the semiclassical asymptotics (corresponding to the double-scaling limit in the matrix model), then the tunneling is exponentially small, and can be neglected. Secondly, and more importantly, the potential does not tend to a constant at infinity, and the effect of the external potential on the asymptotic behavior of the wavefunction does not amount to an $x$-independent phase 
shift. Since the external potential breaks translational invariance even at infinity, one has to label one-particle states by energy $\varepsilon$ rather than momentum. Then at large distances the wavefunction behaves as follows:

$f\left(x_{i}\right) \sim \frac{1}{\sqrt{x}_{i}}\left[\exp \left(-i x_{i}^{2} / 2-i \varepsilon_{i} \log x_{i}-\phi_{-}\left(\varepsilon_{i}\right)\right)-\exp \left(i x_{i}^{2} / 2+i \varepsilon_{i} \log x_{i}+\phi_{b}\left(\varepsilon_{i}\right)+\phi_{+}\left(\varepsilon_{i}\right)\right)\right]$.

Here $\phi_{b}(\varepsilon)$ can be deduced by solving the Schrödinger equation for a single particle in an inverted harmonic potential and is given by [54]:

$$
\phi_{b}(\varepsilon)=\frac{\pi}{2}+\varepsilon \log 2+\arg \Gamma\left(\frac{1}{2}-i \varepsilon\right)
$$

Note that in the derivation of the above equation we have implicitly assumed that the S-matrix factorizes into the product of two-body S-matrices and the boundary S-matrix. This can be justified using the complete integrability of the system.

The Bethe equations now look as follows

$$
L^{2}+2 \varepsilon_{i} \log L+\phi_{b}\left(\varepsilon_{i}\right)+2 \sum_{i \neq j} \phi\left(\varepsilon_{i}-\varepsilon_{j}\right)=2 \pi n_{i}, \quad n_{i} \in \mathbf{Z}
$$

The corresponding integral equation for particle and hole densities is:

$$
2 \pi\left(\rho(\varepsilon)+\rho_{h}(\varepsilon)\right)=2 \log L+\phi_{b}^{\prime}(\varepsilon)+2 \int_{-\infty}^{+\infty} \phi^{\prime}\left(\varepsilon-\varepsilon^{\prime}\right) \rho\left(\varepsilon^{\prime}\right) d \varepsilon^{\prime} .
$$

For the Calogero-Moser interaction potential $U=\frac{l(l-1)}{\left(\lambda_{1}-\lambda_{2}\right)^{2}}$, the two-body phase shift is given by 10 (see e.g. 48 ):

$$
\phi(\varepsilon)=\pi(1-l) \operatorname{sign}(\varepsilon)
$$

Therefore the integral equation for $\rho$ and $\rho_{h}$ becomes a simple algebraic relation:

$$
2 \pi\left(\rho(\varepsilon)+\rho_{h}(\varepsilon)\right)=2 \pi(1-l) \rho(\varepsilon)+2 \log L+\phi_{b}^{\prime}(\varepsilon) .
$$

In other words:

$$
\rho_{h}(\varepsilon)+l \cdot \rho(\varepsilon)=\frac{\log L}{\pi}+\frac{1}{2 \pi} \phi_{b}^{\prime}(\varepsilon) .
$$

The right-hand side of this equation is precisely the one-particle density of states for noninteracting fermions in an inverted harmonic potential (with a wall at $x=L$ ). Note that

10 In this section we set $l=\frac{\alpha}{2}$. 
it is natural to change variables from $x$ to $\tau=\log x$; then $\log L$ can be interpreted as the size of the box in the $\tau$ coordinate. For $l=1$ the equation (4.18) simply says that the sum of the particle and hole densities must add up to the density of available one-particle states. The remarkable feature of the Calogero-Moser potential is that its entire effect is to replace $\rho$ with $l \cdot \rho$.

Put in a more intuitive way, this means that we may treat Calogero-Moser particles as non-interacting and obeying a generalized exclusion principle: the distance between occupied energy levels must be at least $l$ [55] [56] [48]. The cases $l=0$ and $l=1$ correspond to bosons and fermions, respectively. For non-integer $l$ the generalized exclusion principle makes sense only once one averages over many energy levels. As shown in the previous section, the symmetric matrix quantum mechanics model corresponds to $l=1 / 2$, which is half-way between bosons and fermions ("semions"), and is dual to the $\mathrm{O}^{-}$orientifold. The "quaternionic-hermitian" matrix quantum mechanics model corresponds to $l=2$, which means that there should be at least one hole between any two occupied levels ("doublyfermions"), and is dual to the $\mathrm{O1}^{+}$orientifold . To summarize, we can determine all thermodynamic quantities of the Calogero-Moser system in the inverted harmonic potential by treating the particles as non-interacting but obeying generalized exclusion statistics.

Now we are ready to compute the free energy. We express $\rho_{h}$ in terms of $\rho$ and then minimize the functional (4.9). The result is:

$$
F=-T \int_{-\infty}^{\infty} \rho_{0}(\varepsilon) \log \left(1+\frac{n(\varepsilon)}{1-l \cdot n(\varepsilon)}\right) d \varepsilon
$$

Here $\rho_{0}(\varepsilon)$ is the one-particle level-density in the presence of the inverted harmonic potential [54]:

$$
\rho_{0}(\varepsilon)=\frac{1}{\pi} \log (L \sqrt{2})-\frac{1}{2 \pi} \Re \psi\left(\frac{1}{2}-i \varepsilon\right), \quad \psi(u)=\frac{d}{d u} \log \Gamma(u) .
$$

The effective filling factor $n(\varepsilon)$ is determined from the following algebraic equation [56]:

$$
\frac{n(1-(l-1) n)^{l-1}}{(1-l \cdot n)^{l}}=\exp \left(\frac{\mu-\varepsilon}{T}\right)
$$

For $l=1$ the latter equation implies that $n(\varepsilon)$ is the usual Fermi distribution and $F$ is the free energy of free fermions in an inverted harmonic potential:

$$
l=1: \quad n=\frac{1}{e^{\frac{\varepsilon-\mu}{T}}+1}, \quad F=-T \int_{-\infty}^{\infty} \rho_{0}(\varepsilon) \log \left(1+e^{\frac{\mu-\varepsilon}{T}}\right) d \varepsilon
$$


This integral representation for $F$ is divergent, but if we differentiate it with respect to $T$, we get a convergent integral, which can be expanded in powers of $1 / \mu$. This procedure reproduces the temperature-dependent part of the free energy of the Hermitian matrix model [57]:

$$
F(T, \mu)-F(0, \mu)=\frac{\pi T^{2}}{12} \log \mu+O\left(\mu^{-2}\right)
$$

For $l=1 / 2$ ("semions") we get:

$$
l=\frac{1}{2}: \quad n(\varepsilon)=\frac{1}{\sqrt{\frac{1}{4}+\exp \left(\frac{2(\varepsilon-\mu)}{T}\right)}}, \quad F=-T \int_{-\infty}^{\infty} \rho_{0}(\varepsilon) \log \left(\frac{1+\frac{n}{2}}{1-\frac{n}{2}}\right) d \varepsilon
$$

For $l=2$ ("doubly-fermions") we get:

$$
l=2: \quad n(\varepsilon)=\frac{1}{2}\left(1-\frac{1}{\sqrt{1+4 \exp \left(\frac{\mu-\varepsilon}{T}\right)}}\right), \quad F=-T \int_{-\infty}^{\infty} \rho_{0}(\varepsilon) \log \left(\frac{1-n(\varepsilon)}{1-2 n(\varepsilon)}\right) d \varepsilon
$$

The integrals are again divergent, but their temperature-dependent part is convergent. Expanding the integrands in powers of $1 / \mu$, we find:

$$
l=\frac{1}{2}: \quad F(T, \mu)-F(0, \mu)=\frac{\pi T^{2}}{12} \log \mu+\frac{\zeta(3) T^{3}}{4 \pi \mu}+O\left(\mu^{-2}\right)
$$

and

$$
l=2: \quad F(T, \mu)-F(0, \mu)=\frac{\pi T^{2}}{12} \log \mu-\frac{\zeta(3) T^{3}}{2 \pi \mu}+O\left(\mu^{-2}\right) .
$$

Note that the leading-order terms are of order $\log \mu$ and are the same in all three cases $(l=1 / 2,1,2)$. This is to be expected, as the physics can be described in all three cases by a collective field, and to leading order in $1 / N$ the collective field Lagrangians are identical, as shown in the last section. In fact, the $\log \mu$ piece in the free energy is simply the free energy of a massless two dimensional scalar field in a box of length $V_{\phi}=-\frac{1}{2} \log \mu$. This is in agreement with the string theory result (2.15), which says that the only bulk degree of freedom is a massless tachyon. Note also that while for $l=1$ only even powers of $\mu$ appear in the expansion of $F(T, \mu)$, for $l=1 / 2$ and $l=2$ all positive powers of $\mu^{-1}$ contribute. This agrees with the identification of $\mu$ with $1 / g_{s t}$. In paticular, the subleading piece in the temperature-dependent part of $F(T, \mu)$ comes from an unorientable worldsheet with Euler characteristic -1 . 
The temperature-independent part (i.e. the ground state energy) can be determined from:

$$
\frac{\partial E}{\partial \mu}=\mu \rho(\mu)=\frac{1}{l} \mu \rho_{0}(\mu) .
$$

Thus the ground-state energy is simply $1 / l$ times the well-known free-fermion result:

$$
E=F(0, \mu)=\frac{1}{2 \pi l}\left(-\frac{1}{2} \mu^{2} \log \mu+\frac{1}{24} \log \mu-\sum_{m=1}^{\infty} \frac{\left(2^{2 m+1}-1\right)\left|B_{2 m+2}\right|}{8 m(m+1)(2 \mu)^{2 m}}\right) .
$$

\subsection{Particle-hole duality for the Calogero-Moser system}

In the Hermitian matrix quantum mechanics the expansion of the free energy in powers of $1 / \mu$ contains only even powers. From the string theory point of view this follows from the identification of $1 / \mu$ with the string coupling and the fact that only oriented worldsheets contribute. From the free-fermion point of view, this is a consequence of the particle-hole duality, which exchanges $\rho$ and $\rho_{h}$ and takes $\mu$ to $-\mu$. Technically, particle-hole duality holds to all orders in $1 / \mu$ because the one-particle density of states $\rho_{0}(\varepsilon)$ is even.

It turns out that for $l \neq 1$ there is a generalized particle-hole duality which maps the Calogero-Moser system with $l=l_{0}$ into a Calogero-Moser system with $l=1 / l_{0}$. More precisely, we claim that the following relation holds for the free energy of the CalogeroMoser particles in an inverted harmonic potential:

$$
F\left(T, \mu ; l=\frac{1}{l_{0}}\right)=l_{0}^{2} F\left(\frac{T}{l_{0}},-\mu ; l=l_{0}\right)+\text { terms analytic in } \mu
$$

To derive this relation, we define:

$$
\tilde{\rho}=l \cdot \rho_{h}, \quad \tilde{\rho}_{h}=l \cdot \rho, \quad \tilde{l}=\frac{1}{l}, \quad \tilde{T}=l \cdot T, \quad \tilde{\mu}=-\mu .
$$

If $\rho$ and $\rho_{h}$ satisfy $\rho_{h}+l \cdot \rho=\rho_{0}$, then $\tilde{\rho}$ and $\tilde{\rho}_{h}$ satisfy $\tilde{\rho}_{h}+\tilde{l} \cdot \tilde{\rho}=\rho_{0}$. Furthermore, one can easily see that the free energy functional evaluated on the tilded quantities is $l^{2}$ times the free energy functional of the untilded quantities, up to a (divergent) term which is independent of $\rho$ and and $\rho_{h}$ and is linear in $\mu$. This implies the desired result.

For $T=0$ generalized particle-hole duality says that:

$$
E\left(\mu ; l=\frac{1}{l_{0}}\right)=l_{0}^{2} E\left(-\mu ; l=l_{0}\right)+\text { terms analytic in } \mu .
$$

We already know that $E(\mu ; l)$ is $1 / l$ times a function of $\mu$ which is even up to analytic terms. Hence the relation Eq. (4.31) is satisfied. Particle-hole duality also explains why 
the logarithmic terms in Eq.(4.25) and Eq.4.26) are identical, while the terms of order $\mu^{-1}$ differ by a factor -2 .

For $l_{0}=2$ the generalized particle-hole duality has a simple interpretation in terms of dual string theories: it essentially says that the partition functions of the string theories corresponding to $l=1 / 2$ and $l=2$ are related by the substitution $g_{s t} \rightarrow-g_{s t}$, i.e. by changing the sign of the contribution from worldsheets with odd Euler characteristic. To see this more clearly, we first rewrite the relation Eq. (4.29) in terms of the partition functions of the Calogero-Moser systems:

$$
\log Z_{C M}(T, \mu ; l=1 / 2)=2 \log Z_{C M}\left(\frac{T}{2},-\mu ; l=2\right)+\text { terms analytic in } \mu \text {. }
$$

Next we note that because in the quaternionic-Hermitian case each eigenvalue of $M$ appears twice, the Hamiltonian of the quaternionic-Hermitian matrix quantum mechanics is twice the Hamiltonian of the corresponding Calogero-Moser system. This implies that the definitions of temperature in the Calogero-Moser system and the matrix quantum mechanics differ by a factor of two. Hence the partition functions of the matrix quantum mechanics are related as follows:

$$
\log Z_{M Q M}^{S O}(T, \mu)=2 \log Z_{M Q M}^{S p}(T,-\mu)+\text { terms analytic in } \mu .
$$

On the other hand, the partition functions in string theory satisfy

$$
Z_{s t}^{S O}\left(T, g_{s t}\right)=Z_{s t}^{S p}\left(T,-g_{s t}\right)
$$

This equation agrees with Eq. (4.33) if we identify $Z_{s t}^{S O}$ with $\log Z_{M Q M}^{S O}$ and $Z_{s t}^{S p}$ with $2 \cdot \log Z_{M Q M}^{S p}$. The normalization factor 2 in the last equation is needed to make the leading term in the temperature-dependent part of $F_{M Q M}^{S p}(T, \mu)$ be equal to the free energy of a massless two-dimensional scalar field in a box of length $-\frac{1}{2} \log \mu$.

\section{Unoriented $\hat{c}=1$ Type 0B Strings}

The worldsheet description of $D=2$ superstring theory is given by a free $\mathcal{N}=1$ superfield tensored with $\mathcal{N}=1$ super-Liouville with $\hat{c}_{L}=9 . \mathcal{N}=1$ super-Liouville is 
defined by an $\mathcal{N}=1$ superfield $\Phi$ with components $(\phi, \psi)$ and a superpotential $W=\mu e^{b \Phi}$. The GSO projection giving rise to Type 0B string theory is given by:

$$
\begin{aligned}
\text { NS-NS }: & \frac{1}{2}\left(1+(-1)^{F+\tilde{F}}\right) \\
\text { RR }: & \frac{1}{2}\left(1+(-1)^{F+\tilde{F}}\right) .
\end{aligned}
$$

The spectrum of the model consists of a massless tachyon $T$ arising form the NS-NS sector and an additional massless scalar $C$ arising from the $\mathrm{RR}$ sector.

Due to the non-chiral GSO projection, the theory is manifestly invariant under the action of worldsheet parity $\Omega$. We define the action of $\Omega$ on the fermions and on the spin fields as follows:

$$
\begin{aligned}
& \Omega \cdot \psi=\bar{\psi}, \Omega \cdot \bar{\psi}=-\psi \Longrightarrow \Omega \cdot(\bar{\psi} \psi)=\bar{\psi} \psi, \\
& \Omega \cdot S^{\alpha}=\bar{S}^{\alpha}, \Omega \cdot \bar{S}^{\alpha}=S^{\alpha} \Longrightarrow \Omega \cdot\left(\bar{S}^{\alpha} \otimes S^{\beta}\right)=-\bar{S}^{\beta} \otimes S^{\alpha} .
\end{aligned}
$$

The action of $\Omega$ can be combined with the action of other $\mathbf{Z}_{2}$ symmetries to yield other consistent orientifold projections. The theory is invariant under $(-1)^{F_{s}^{L}}$, where $F_{s}^{L}$ is left-moving space-time fermion number, so that one can consider modding out the theory by the following two orientifold groups 11 :

1) $G_{1}=\{1, \Omega\}$

2) $G_{2}=\left\{1, \Omega(-1)^{F_{s}^{L}}\right\}$.

We now analyze the physics of these two models.

- Modding Type $0 B$ by $G_{1}=\{1, \Omega\}$

Given the action of $\Omega$ in $(\underline{5.2})$ it follows that the only state surviving the projection is $T$. Therefore the space-time physics of this orientifold model is described by a massless scalar field $T$.

To understand better the spectrum of the model we now compute the partition function, which is given by:

$$
Z=\operatorname{Tr}_{N S-N S \oplus R R}\left[\left(\frac{1+\Omega}{2}\right)\left(\frac{1+(-1)^{F+\tilde{F}}}{2}\right) q^{L_{0}} \bar{q}^{\bar{L}_{0}}\right]=\frac{1}{2} Z_{T^{2}}+\frac{1}{2} Z_{K B} .
$$

11 In ten dimensions one can also consider the model (see e.g. [58] [59]) obtained by modding out by the orientifold group $G=\left\{1, \Omega(-1)^{F}\right\}$. In $D=2,(-1)^{F}$ is not a symmetry since it flips the sign of the term $\bar{\psi} \psi e^{b \phi}$ in the super-Liouville action. 
The torus contribution has recently been computed [1] and is given by

$$
Z_{T^{2}}=V_{\phi} \frac{1}{12}\left(\frac{R}{\sqrt{\alpha^{\prime}}}+2 \frac{\sqrt{\alpha^{\prime}}}{R}\right)
$$

where $V_{\phi}$ is the volume of the Liouville direction.

In computing the Klein bottle contribution we note that the contribution from $\Omega$-even states, which are the only states contributing to the trace, are automatically $(-1)^{F+\tilde{F}}$-even. Therefore we only need to compute

$$
Z_{K B}=\operatorname{Tr}_{N S-N S \oplus R R}\left[\Omega q^{L_{0}} \bar{q}^{\bar{L}_{0}}\right]
$$

Now we note that the oscillator contribution from the free superfield and super-Liouville are precisely cancelled by the contribution from the ghosts and superghosts determinants in each sector. Therefore, the Klein bottle is determined by the action of the orientifold group on the zero modes. Combining (2.12) with the fact that $\Omega \cdot|0\rangle_{N S-N S}=|0\rangle_{N S-N S}$, it follows that the Klein bottle contribution vanishes. Therefore, the partition function of the model is given by

$$
Z=\frac{1}{2} Z_{T^{2}}=V_{\phi} \frac{1}{24}\left(\frac{R}{\sqrt{\alpha^{\prime}}}+2 \frac{\sqrt{\alpha^{\prime}}}{R}\right)
$$

whose temperature dependent contribution is precisely that of a massless two-dimensional field, as expected from space-time considerations.

One can extract further information about the contribution from unoriented surfaces by analyzing the crosscap state $|C\rangle$, which describes the closed string state produced by adding a crosscap to a worldsheet. $|C\rangle$ must be invariant under the gauge symmetries of the model, in particular it has to be GSO invariant. Moreover, it must reproduce the Klein bottle loop amplitude (5.4) when factorizing the computation of its norm 12 into the loop channel:

$$
\left\langle C\left|e^{-l H_{c}}\right| C\right\rangle=\operatorname{Tr}_{N S-N S \oplus R R}\left[\Omega\left(\frac{1+(-1)^{F+\tilde{F}}}{2}\right) q^{L_{0}} \bar{q}^{\bar{L}_{0}}\right] .
$$

Since the right-hand side vanishes, so does the left-hand side. Inserting a complete set of physical states, we see that the overlap (regularized by $e^{-l H_{c}}$ ) of the crosscap state with any physical state must vanish.

12 When computing the norm one must insert the damping factor $e^{-l H_{c}}$. 
One can see the vanishing of the overlap of the crosscap with the physical states more explicitly as follows. By analyzing the effect of the twists when factorizing from the loop channel to the tree channel, one finds that $|C\rangle$ only has contributions from the NS-NS sector

$$
|C\rangle=\frac{1}{\sqrt{2}}(|N S N S,+\rangle-|N S N S,-\rangle),
$$

where the signs \pm label the spin structure on $R P^{2}$, and $|N S N S\rangle$ solves the crosscap conditions [38] [39]. The absence of the RR contribution is to be expected, as there are no physical states in the RR sector. In the NS-NS sector the only physical state is the tachyon, which is the ground state. Since $|N S N S,+\rangle$ and $|N S N S,-\rangle$ differ only by oscillator terms, it follows that the projection of $|C\rangle$ on the tachyon state vanishes.

The vanishing of the overlap between the crosscap and the physical states implies that the contribution of all unorientable worldsheets to the S-matrix also vanishes. At first sight, this seems rather strange. However, we will see that this is precisely what the matrix model predicts. Moreover, it predicts that the tachyon S-matrix in this model is equal to the tachyon S-matrix of the oriented $\hat{c}=1$ Type $0 \mathrm{~B}$ string theory.

- Modding Type $0 B$ by $G_{2}=\left\{1, \Omega(-1)^{F_{s}^{L}}\right\}$

The effect of the extra $(-1)^{F_{s}^{L}}$ projection results in important differences in the spectrum. In the NS-NS sector $(-1)^{F_{s}^{L}}$ acts trivially, so that $T$ again survives the projection. In the RR sector $(-1)^{F_{s}^{L}}$ acts with an extra minus sign so that $C$ is now invariant under the orientifold projection, and the spectrum is the same as in Type $0 \mathrm{~B}$ oriented string theory. The dynamics, however, is rather different.

We now proceed to compute 13 the partition function of the model, which can be obtained from (5.4) by substituting $\Omega \rightarrow \Omega(-1)^{F_{s}^{L}}$. The toroidal contribution is the same and is given by (5.7). The Klein bottle contribution is very different since now both the NS-NS and RR ground states are even under $\Omega(-1)^{F_{s}}$, so that their contributions add instead of cancelling each other as in the previous model. Therefore, the crosscap state for this orientifold model is given by:

$$
|C\rangle=\frac{1}{\sqrt{2}}(|N S N S,+\rangle+|N S N S,-\rangle) .
$$

13 We will be rather brief in this section since up to some important minus signs the computations are the same as in the previous model. 
It follows that the Klein bottle contribution for this orientifold is twice that of the bosonic orientifold (2.14). As in the bosonic string orientifold, the model has a divergence as a result of a massless tachyon tadpole on the $R P^{2}$ worldsheet, which is the same as in the bosonic model up to an extra factor of $\sqrt{2}$. We cancel the Klein bottle divergence via the Fischler-Susskind mechanism as in the bosonic model by adding to the worldsheet a counterterm proportional to the super-Liouville interaction operator. After cancelling this divergence the resulting partition function is:

$$
Z^{r e n}=V_{\phi} \frac{1}{24}\left(\frac{R}{\sqrt{\alpha^{\prime}}}+4 \frac{\sqrt{\alpha^{\prime}}}{R}\right)
$$

We note that the temperature dependent contribution is that of two massless twodimensional fields, as expected from space-time considerations.

\section{Unoriented $\hat{c}=1$ OB Matrix Model}

Following the discussion in section 3 , it is natural to conjecture that the matrix model description of the Type 0B orientifold models is given by the worldvolume action of $N$ unstable D0-branes.

The spectrum of open strings on D0-branes in Type 0B string theory is the same [60] 61] as in the bosonic string, that is, there is a tachyon mode $M$ and a non-dynamical gauge field $A$. As in section 3 , in order to determine the matrix model dual for the Type 0B orientifolds, we must understand the action of the orientifold group on the open string spectrum. The vertex operators for the tachyon and gauge field in Type 0B string theory contain extra fermions compared to the vertex operators in the bosonic string, which result in different projections when modding out by the orientifold group.

- Modding Type $0 B$ by $G_{1}=\{1, \Omega\}$

When computing the action of $\Omega$ on the open string tachyon state, one finds that it is odd 14. Therefore, the worldvolume spectrum for this orientifold model is given by

14 The transformation properties of the tachyon can be extracted from the non-vanishing of the disk amplitude with a RR vertex operator and the open string tachyon and using the known transformation properties of the RR vertex operator under various symmetries. 
(depending on the sign of the crosscap state)

$$
\begin{aligned}
& O 1^{-}: M^{t}=-M \quad A^{t}=-A \\
& O 1^{+}: J M^{t} J=M \quad J A^{t} J=A,
\end{aligned}
$$

where $J$ is the usual $S p(N)$ invariant tensor. For this orientifold projection, both the open string tachyon and the gauge field belong to the $S O(N)$ or $S p(N)$ Lie algebra. As before, the equation of motion for $A$ just forces one to consider states in the singlet sector.

Therefore, the matrix model dual to this orientifold model is given by (3.2) with the matrices belonging to the $S O(N)$ or $S p(N)$ Lie algebra and with the potential for the tachyon now being even, that is $U(x)=U(-x)$. In order to analyze these matrix models, we go to the eigenvalue basis. As in section 3 we must compute the Jacobian of the change of coordinates. By using the fact that the generators of the $S O(N)$ Lie algebra are antisymmetric and those of $S p(N)$ are symmetric, one finds that the Jacobian factors are given by 62 :

$$
\begin{aligned}
& O 1^{-}: J=\prod_{i<j}\left(\lambda_{i}-\lambda_{j}\right)^{2}\left(\lambda_{i}+\lambda_{j}\right)^{2} \\
& O 1^{+}: J=\prod_{i} \lambda_{i}^{2} \prod_{i<j}\left(\lambda_{i}-\lambda_{j}\right)^{2}\left(\lambda_{i}+\lambda_{j}\right)^{2} .
\end{aligned}
$$

Therefore, the Schrödinger equation that needs to be solved - in the singlet sector - is given by (3.4). As in section 3 , we redefine the wavefunction $\Psi(\lambda)=\operatorname{sign}(J)|J|^{-1 / 2} f(\lambda)$ to eliminate terms with a single derivative. It is now a straightforward computation to show that $f(\lambda)$ is an eigenfunction of the following Hamiltonian:

$$
\tilde{H}=-\frac{1}{2} \sum_{i} \frac{d^{2}}{d \lambda_{i}^{2}}+\sum_{i} U\left(\lambda_{i}\right) .
$$

Therefore, the solution of this matrix model reduces to analyzing the dynamics of $N$ free fermions in an inverted harmonic oscillator potential. This is identical to the result that one gets when one rewrites the Hermitian matrix model in terms of its eigenvalues. The only difference compared to that case is that now wavefunctions are either even or odd depending on the choice of the orientifold projection:

$$
\begin{aligned}
& O 1^{-}: f\left(-\lambda_{i}\right)=f\left(\lambda_{i}\right) \\
& O 1^{+}: f\left(-\lambda_{i}\right)=-f\left(\lambda_{i}\right) .
\end{aligned}
$$

Therefore scattering amplitudes for the $S O(N)$ and $S p(N)$ Lie algebra valued matrix models are exactly the same as for the tachyon in the $\hat{c}=1$ oriented Type $0 \mathrm{~B}$ string 
theory. As for the free energy, it is half the free energy of the $\hat{c}=1$ oriented Type 0B string.

This fits perfectly with the conjectured string theory dual, Type 0B string theory modded out by $G_{1}=\{1, \Omega\}$. As discussed in section 4 , this string theory has only contributions from oriented Riemann surfaces. Moreover, we showed that the free energy is one half that for oriented $\hat{c}=1$ Type $0 \mathrm{~B}$ string theory.

- Modding Type $0 B$ by $G_{2}=\left\{1, \Omega(-1)^{F_{s}^{L}}\right\}$

As before, the matrix model is obtained by projecting the open string spectrum by $G_{2}$. The open string tachyon on the D0-branes is separately odd under $\Omega$ and $(-1)^{F_{s}^{L}}$. Therefore, the worldvolume spectrum for this orientifold model is given by:

$$
\begin{aligned}
& O 1^{-}: M^{t}=M \quad A^{t}=-A \\
& O 1^{+}: J M^{t} J=-M \quad J A^{t} J=A .
\end{aligned}
$$

This is precisely the same spectrum as for the $c=1$ bosonic string orientifold.

As shown in section 3, the dynamics of this matrix model is that of fermions in an inverted harmonic potential interacting via the Calogero-Moser potential (3.5). The only difference in solving this model compared to its bosonic string analog is that when constructing the quasi-Fermi sea for the dual of the Type 0B orientifold one fills the states symmetrically with respect to the tachyon maximum, due to the $\mathbf{Z}_{2}$ symmetry of the open string tachyon potential [10] [1] . Therefore the free energy in this case should be twice that of the bosonic string, in agreement with the computation in section 4. Further, all tachyon scattering amplitudes coincide with those for the bosonic string to all orders in perturbation theory.

\section{Unoriented $\hat{c}=1$ Type 0A Strings}

The worldsheet CFT is the same as for two-dimensional Type 0B string theory. The GSO projection is different and is given by:

$$
\begin{aligned}
\text { NS-NS }: & \frac{1}{2}\left(1+(-1)^{F+\tilde{F}}\right) \\
\mathrm{RR} & : \frac{1}{2}\left(1-(-1)^{F+\tilde{F}}\right) .
\end{aligned}
$$


The spectrum of the model is a massless tachyon $T$ arising form the NS-NS sector and two non-dynamical gauge fields $C_{+}$and $C_{-}$arising from the RR sector. More precisely, the gauge fields can be written as $C_{ \pm}=\frac{1}{\sqrt{2}}(C \pm \tilde{C})$, where $C, \tilde{C}$ are the vacuum states of the $(R+, R-),(R-, R+)$ sectors respectively. The model has stable D0-branes which couple 15 to $C_{-}$; they play an important role when deriving the matrix model dual.

The Type 0A GSO projection is non-chiral, so we can construct new models by modding out by the allowed orientifold groups $G$. The symmetries are the same as in Type 0B. Therefore we can quotient the theory by the orientifold groups $G_{1}$ or $G_{2}$ in (5.3).

- Modding Type $0 A$ by $G_{1}=\{1, \Omega\}$

The tachyon mode is invariant under the action of $\Omega$. In the RR sector $\Omega$ acts by identifying the two RR sectors:

$$
\Omega \cdot C=-\tilde{C} \quad \Omega \cdot \tilde{C}=-C .
$$

Therefore, only $T$ and $C_{-}$survive the orientifold projection.

The partition function of the model is given by:

$$
\begin{aligned}
Z & =\operatorname{Tr}_{N S-N S}\left[\left(\frac{1+\Omega}{2}\right)\left(\frac{1+(-1)^{F+\tilde{F}}}{2}\right) q^{L_{0}} \bar{q}^{\bar{L}_{0}}\right]+\operatorname{Tr}_{R R}\left[\left(\frac{1+\Omega}{2}\right)\left(\frac{1-(-1)^{F+\tilde{F}}}{2}\right) q^{L_{0}} \bar{q}^{\bar{L}_{0}}\right] \\
& =\frac{1}{2} Z_{T^{2}}+\frac{1}{2} Z_{K B} .
\end{aligned}
$$

The torus amplitude has been computed in [11] and is given by:

$$
Z_{T^{2}}=V_{\phi} \frac{1}{12}\left(\frac{2 R}{\sqrt{\alpha^{\prime}}}+\frac{\sqrt{\alpha^{\prime}}}{R}\right) \text {. }
$$

A simple consequence of the action of $\Omega$ on the RR sectors (7.2) is that the RR contribution to the Klein bottle vanishes. Since the action is off-diagonal, the RR states do not contribute to the trace. Therefore, the Klein bottle is given precisely by (2.14) and it has a divergent contribution arising from a tachyon tadpole on $R P^{2}$. This can be easily shown by analyzing the crosscap state for this model, which is given by

$$
|C\rangle=|N S N S,+\rangle
$$

15 The other gauge field $C_{+}$, has no physical D0-brane which couples to it 11. 
and has a non-trivial coupling to the zero momentum tachyon. After cancelling the divergence due to the tachyon tadpole via the Fischler-Susskind mechanism as explained in section 2 , the partition function is given by:

$$
Z^{r e n}=V_{\phi} \frac{1}{24}\left(\frac{2 R}{\sqrt{\alpha^{\prime}}}+\frac{\sqrt{\alpha^{\prime}}}{R}\right)
$$

The temperature dependent piece is given by the contribution of a two-dimensional massless scalar field, as expected from space-time considerations.

- Modding Type $0 A$ by $G_{2}=\left\{1, \Omega(-1)^{F_{s}^{L}}\right\}$

The action of $(-1)^{F_{s}^{L}}$ yields an extra minus sign in the RR sector compared to (7.2). Therefore, for this model the states invariant under the orientifold projection are $T$ and $C_{+}$.

The partition function of the model is given by (7.3) with the replacement $\Omega \rightarrow$ $\Omega(-1)^{F_{s}^{L}}$. Since $(-1)^{F_{s}^{L}}$ acts trivially in the NS-NS sector, the contribution from this sector to the Klein bottle is the same as in the previous orientifold. Moreover, the RR sector contribution to the Klein bottle also vanishes, due again to the non-diagonal action of $\Omega(-1)^{F_{s}^{L}}$. Therefore, both the crosscap state and the renormalized partition function are the same as in the previous model and are given by (7.5) (7.6).

Despite many similarities, the fact that $C_{+}$is invariant as opposed to $C_{-}$will result in important differences in constructing the matrix model dual.

\section{Unoriented $\hat{c}=1$ 0A Matrix Model}

The strategy here is to analyze the worldvolume theory on an unstable D0-brane system. It is important to consider a brane configuration that is unstable 16 , so that it has an open string tachyon in its spectrum, and we can recover the original closed string physics after tachyon condensation.

16 The theory also has uncharged D1-branes, but they are stable, because the open string "tachyon" is actually massless. 
The open string spectrum on a collection of D0-branes is stable in Type 0A string theory. The D0-branes carry charge under $C_{-}$, which can be read off from the boundary state:

$$
|D 0\rangle=\frac{1}{\sqrt{2}}(|N S N S,-\rangle+|R R,-\rangle) .
$$

The presence of the RR piece in the boundary state is responsible for projecting out the open string tachyon present in the bosonic string (and Type 0B string). This can be shown by factorizing the cylinder amplitude into the annulus, which yields a GSO projected open string partition function:

$$
Z=\operatorname{Tr}_{N S \oplus R}\left(\frac{1+(-1)^{F}}{2} e^{-t H_{o}}\right) .
$$

Therefore, we must consider a D0 $-\overline{\mathrm{D} 0}$ system in order to construct the dual matrix model. As in [11], we will study a collection of $N$ D0-branes and $N+m \overline{\mathrm{D} 0}$-branes. As usual, the GSO projection for $\mathrm{D} 0-\overline{\mathrm{D} 0}$ open strings is reversed compared to that for D0-D0 open strings so that a tachyon appears in the spectrum. The effective theory [10] [11] is described by a matrix quantum mechanics with a $U(N) \times U(N+m)$ gauge field and a complex tachyon in the bifundamental representation:

$$
A=\left(\begin{array}{cc}
A_{1} & 0 \\
0 & A_{2}
\end{array}\right) \quad M=\left(\begin{array}{cc}
0 & t \\
t^{\dagger} & 0
\end{array}\right) .
$$

After gauge-fixing, the problem reduces to studying the dynamics of $N$ non-interacting fermions moving in a plane with angular momentum $m$ and subject to an inverted harmonic oscillator potential [11].

We now consider the orientifold models.

- Modding Type $0 A$ by $G_{1}=\{1, \Omega\}$

Since $C_{-}$is invariant under the action of $\Omega$, branes are mapped to branes, and antibranes to anti-branes, under this symmetry. This means that we can mode out by $G_{1}$ an arbitrary D0 $-\overline{\mathrm{D} 0}$ configuration (i.e. $m$ can be arbitrary).

Demanding that the open string vertex operators are invariant under the action of $g=\Omega$ results in the following projections:

$$
\begin{aligned}
\gamma(g) A^{t} \gamma(g)^{-1} & =-A, \\
\gamma(g) M^{t} \gamma(g)^{-1} & =M,
\end{aligned}
$$


where $A$ and $M$ are given in (8.3). There are two choices for the representation matrices $\gamma(g)$, depending on the type of orientifold. They are given by:

$$
\begin{aligned}
& O 1^{-}: \gamma(g)=\left(\begin{array}{ll}
I & 0 \\
0 & I
\end{array}\right) \\
& O 1^{+}: \gamma(g)=\left(\begin{array}{cc}
\left(\begin{array}{cc}
0 & i I \\
-i I & 0
\end{array}\right) & 0 \\
0 & \left(\begin{array}{cc}
0 & i I \\
-i I & 0
\end{array}\right)
\end{array}\right)
\end{aligned}
$$

The solution to (8.4) for the gauge field yields

$$
\begin{aligned}
& O 1^{-}: A \text { is in the } S O(N) \times S O(N+m) \text { Lie algebra } \\
& O 1^{+}: A \text { is in the } S p(N) \times S p(N+m) \text { Lie algebra, }
\end{aligned}
$$

while the solution for $M$ yields a field $t$ in the bifundamental representation of the corresponding gauge group satisfying the following reality conditions:

$$
\begin{aligned}
& O 1^{-}: t=t^{*}, \\
& O 1^{+}: t=-J t^{*} J,
\end{aligned}
$$

where $J$ is the canonical $S p(N)$ invariant tensor.

- Modding Type $0 A$ by $G_{2}=\left\{1, \Omega(-1)^{F_{s}^{L}}\right\}$

We already saw in the previous section that $C_{-}$is odd under the action of $g=$ $\Omega(-1)^{F_{s}^{L}}$. This means that a Type 0A D0-brane gets mapped to a $\overline{\mathrm{D} 0}$-brane under the action of $g$. Therefore, in order to be able to mod out Type $0 \mathrm{~A}$ by $G_{2}$ in the presence of a $\mathrm{D} 0-\overline{\mathrm{D} 0}$ system, we must demand that $m=0$, so that there is an equal number of branes and anti-branes and the configuration is $g$-symmetric.

Since $g$ acts by mapping 00-strings to $\overline{0} \overline{0}$-strings, the two factors in the $U(N) \times U(N)$ gauge group are identified, and we are left with a $U(N)$ gauge field. We now analyze the effect of the projection on $M$ (8.3). There are two inequivalent choices of how to project the open string spectrum, depending on whether the representation matrix $\gamma(g)$ acting on the Chan-Paton indices is symmetric or antisymmetric. The resulting models are:

$$
\begin{array}{ll}
O 1^{-}: \gamma(g)=\left(\begin{array}{cc}
0 & I \\
I & 0
\end{array}\right) & \gamma(g) M^{t} \gamma(g)^{-1}=M \Longrightarrow t \text { symmetric } \\
O 1^{+}: \gamma(g)=\left(\begin{array}{cc}
0 & i I \\
-i I & 0
\end{array}\right) & \gamma(g) M^{t} \gamma(g)^{-1}=M \Longrightarrow t \text { antisymmetric. }
\end{array}
$$


By solving the projection we find that the matrix model dual to the this orientifold model is a $U(N)$ gauge field together with a complex symmetric or antisymmetric matrix $t$, corresponding respectively to the model with an $\mathrm{O1}^{-}$or $\mathrm{O1}^{+}$orientifold.

Next we perform the gauge-fixing procedure for these matrix models. We start with the rectangular (or quiver) matrix models dual to the orientifold projections by $G_{1}$. Using the action of the gauge symmetry, one can bring the $N \times(N+m)$ rectangular matrix $M$ to an $N \times N$ diagonal matrix with the rest of entries vanishing. One can then compute the Jacobian for the change of coordinates. The result is 63 664:

$$
\begin{aligned}
U(N) \times U(N+m): & J=\prod_{i} \lambda_{i}^{1+2 m} \prod_{k<l}\left(\lambda_{k}^{2}-\lambda_{l}^{2}\right)^{2} \\
S O(N) \times S O(N+m) & : J=\prod_{i} \lambda_{i}^{m} \prod_{k<l}\left(\lambda_{k}^{2}-\lambda_{l}^{2}\right) \\
S p(N) \times S p(N+m): & J=\prod_{i} \lambda_{i}^{4 m+3} \prod_{k<l}\left(\lambda_{k}^{2}-\lambda_{l}^{2}\right)^{4} .
\end{aligned}
$$

The Schrödinger equation that needs to be solved is (3.4). By redefining the wavefunction $\Psi(\lambda)=\operatorname{sign}(J) J^{-1 / 2} f(\lambda)$, we find that $f(\lambda)$ is an eigenfunction of the following Hamiltonian:

$$
\begin{aligned}
\tilde{H} & =-\frac{1}{2} \sum_{i} \frac{d^{2}}{d \lambda_{i}^{2}}+\alpha\left(\frac{\alpha}{2}-1\right) \sum_{i<j}\left(\frac{1}{\left(\lambda_{i}-\lambda_{j}\right)^{2}}+\frac{1}{\left(\lambda_{i}+\lambda_{j}\right)^{2}}\right) \\
& +\frac{\alpha}{2}(m+b)\left(\frac{\alpha}{2}(m+b)-1\right) \sum_{i} \frac{1}{\lambda_{i}^{2}}+\sum_{i} U\left(\lambda_{i}\right) .
\end{aligned}
$$

The parameters $\alpha$ and $b$ depend on the model and are displayed in Table 1. This Hamiltonian describes the so-called $B C_{N}$ Calogero-Moser system. Its complete integrability on the quantum level has been demonstrated in 650 [66]. It should be possible to determine its free energy in the double-scaling limit using the asymptotic Bethe ansatz; we leave this as a problem for the future.

\begin{tabular}{|c|c|c|c|}
\hline & $U$ & $S O$ & $S p$ \\
\hline$\alpha$ & 2 & 1 & 4 \\
\hline$b$ & $1 / 2$ & 0 & $3 / 4$ \\
\hline
\end{tabular}

Table 1: Values of parameters for the various models. 
One can analyze the matrix model for the orientifold of Type $0 \mathrm{~A}$ by $G_{2}$ in a similar way. The gauge group is $U(N)$, and the tachyon field $M$ is a symmetric or antisymmetric complex matrix. Reducing to the eigenvalues, we find the following Jacobians:

$$
\begin{gathered}
M^{t}=M: J=\prod_{i} \lambda_{i} \prod_{k<l}\left(\lambda_{k}^{2}-\lambda_{l}^{2}\right), \\
M^{t}=-M: J=\prod_{i} \lambda_{i} \prod_{k<l}\left(\lambda_{k}^{2}-\lambda_{l}^{2}\right)^{4} .
\end{gathered}
$$

The corresponding Hamiltonian is given by Eq. (8.10) with $m=0, \alpha=1, b=1$ (for $M^{t}=M$ ) and $m=0, \alpha=4, b=1 / 4$ (for $M^{t}=-M$ ). In particular, the quantum mechanics of the eigenvalues is integrable.

\section{Acknowledgements}

We would like to thank Juan Maldacena for useful discussions. J. G. was supported by the Sherman Fairchild Prize Fellowship. This research was supported in part by the DOE grant DE-FG03-92-ER40701. 


\section{References}

[1] I. R. Klebanov, "String theory in two-dimensions," arXiv:hep-th/9108019.

[2] P. Ginsparg and G. W. Moore, "Lectures On 2-D Gravity And 2-D String Theory," arXiv:hep-th/9304011.

[3] A. Jevicki, "Development in 2-d string theory," arXiv:hep-th/9309115.

[4] J. Polchinski, "What is string theory?," arXiv:hep-th/9411028.

[5] J. McGreevy and H. Verlinde, "Strings from tachyons: The $\mathrm{c}=1$ matrix reloated," arXiv:hep-th/0304224.

[6] I. R. Klebanov, J. Maldacena and N. Seiberg, "D-brane decay in two-dimensional string theory," JHEP 0307, 045 (2003) arXiv:hep-th/0305159.

[7] J. McGreevy, J. Teschner and H. Verlinde, "Classical and quantum D-branes in 2D string theory," arXiv:hep-th/0305194.

[8] A. Sen, "Descent relations among bosonic D-branes," Int. J. Mod. Phys. A 14, 4061 (1999) arXiv:hep-th/9902105.

[9] A. Sen, "Non-BPS states and branes in string theory," arXiv:hep-th/9904207.

[10] T. Takayanagi and N. Toumbas, "A matrix model dual of type 0B string theory in two dimensions," JHEP 0307, 064 (2003) arXiv:hep-th/0307083.

[11] M. R. Douglas, I. R. Klebanov, D. Kutasov, J. Maldacena, E. Martinec and N. Seiberg, "A new hat for the c $=1$ matrix model," arXiv:hep-th/0307195.

[12] E. J. Martinec, "The annular report on non-critical string theory," arXiv:hepth/0305148.

[13] S. Y. Alexandrov, V. A. Kazakov and D. Kutasov, "Non-perturbative effects in matrix models and D-branes," JHEP 0309, 057 (2003) arXiv:hep-th/0306177].

[14] D. Gaiotto, N. Itzhaki and L. Rastelli, "On the BCFT description of holes in the $\mathrm{c}=$ 1 matrix model," arXiv:hep-th/0307221.

[15] M. Gutperle and P. Kraus, "D-brane dynamics in the $\mathrm{c}=1$ matrix model," arXiv:hepth/0308047.

[16] A. Sen, "Open-closed duality: Lessons from matrix model," arXiv:hep-th/0308068.

[17] J. McGreevy, S. Murthy and H. Verlinde, "Two-dimensional superstrings and the supersymmetric matrix model," arXiv:hep-th/0308105.

[18] A. Kapustin, "Noncritical superstrings in a Ramond-Ramond background," arXiv:hepth/0308119.

[19] J. Teschner, "On boundary perturbations in Liouville theory and brane dynamics in noncritical string theories," arXiv:hep-th/0308140.

[20] A. Giveon, A. Konechny, A. Pakman and A. Sever, "Type 0 strings in a 2-d black hole," arXiv:hep-th/0309056.

[21] J. L. Karczmarek and A. Strominger, "Matrix cosmology," arXiv:hep-th/0309138. 
[22] O. DeWolfe, R. Roiban, M. Spradlin, A. Volovich and J. Walcher, "On the S-matrix of type 0 string theory," arXiv:hep-th/0309148.

[23] I. R. Klebanov, J. Maldacena and N. Seiberg, "Unitary and complex matrix models as 1-d type 0 strings," arXiv:hep-th/0309168.

[24] S. Dasgupta and T. Dasgupta, "Renormalization group approach to $\mathrm{c}=1$ matrix model on a circle and D-brane decay," arXiv:hep-th/0310106.

[25] S. Alexandrov, " $(\mathrm{m}, \mathrm{n}) \mathrm{ZZ}$ branes and the $\mathrm{c}=1$ matrix model," arXiv:hep-th/0310135.

[26] S. H. Shenker, "The Strength Of Nonperturbative Effects In String Theory," RU-9047 Presented at the Cargese Workshop on Random Surfaces, Quantum Gravity and Strings, Cargese, France, May 28 - Jun 1, 1990

[27] O. Aharony, M. Berkooz and E. Silverstein, "Multiple-trace operators and non-local string theories," JHEP 0108, 006 (2001) arXiv:hep-th/0105309.

[28] L. Susskind, "The anthropic landscape of string theory," arXiv:hep-th/0302219.

[29] M. R. Douglas, "The statistics of string / M theory vacua," JHEP 0305, 046 (2003) arXiv:hep-th/0303194.

[30] W. Fischler and L. Susskind, "Dilaton Tadpoles, String Condensates And Scale Invariance," Phys. Lett. B 171, 383 (1986).

[31] W. Fischler and L. Susskind, "Dilaton Tadpoles, String Condensates And Scale Invariance. 2," Phys. Lett. B 173, 262 (1986).

[32] S. R. Das, A. Dhar, A. M. Sengupta and S. R. Wadia, "New Critical Behavior In D = 0 Large N Matrix Models," Mod. Phys. Lett. A 5, 1041 (1990).

[33] S. S. Gubser and I. R. Klebanov, "A Modified c $=1$ matrix model with new critical behavior," Phys. Lett. B 340, 35 (1994) arXiv:hep-th/9407014.

[34] H. Dorn and H. J. Otto, "On correlation functions for noncritical strings with c $\mathrm{i}=1$ d $i=1, "$ Phys. Lett. B 291, 39 (1992) arXiv:hep-th/9206053.

[35] H. Dorn and H. J. Otto, "Two and three point functions in Liouville theory," Nucl. Phys. B 429, 375 (1994) arXiv:hep-th/9403141.

[36] A. B. Zamolodchikov and A. B. Zamolodchikov, "Structure constants and conformal bootstrap in Liouville field theory," Nucl. Phys. B 477, 577 (1996) arXiv:hepth/9506136].

[37] J. Teschner, "Liouville theory revisited," Class. Quant. Grav. 18, R153 (2001) arXiv:hep-th/0104158.

[38] C. G. Callan, C. Lovelace, C. R. Nappi and S. A. Yost, "Adding Holes And Crosscaps To The Superstring," Nucl. Phys. B 293, 83 (1987).

[39] J. Polchinski and Y. Cai, "Consistency Of Open Superstring Theories," Nucl. Phys. B 296, 91 (1988).

[40] Y. Hikida, JHEP 0305, 002 (2003) arXiv:hep-th/0210305. 
[41] P. Di Francesco and D. Kutasov, "World sheet and space-time physics in twodimensional (Super)string theory," Nucl. Phys. B 375, 119 (1992) arXiv:hepth/9109005.

[42] A. Gupta, S. P. Trivedi and M. B. Wise, "Random Surfaces In Conformal Gauge," Nucl. Phys. B 340, 475 (1990).

[43] M. Bershadsky and I. R. Klebanov, "Genus One Path Integral In Two-Dimensional Quantum Gravity," Phys. Rev. Lett. 65, 3088 (1990).

[44] Y. Nakayama, "Tadpole cancellation in unoriented Liouville theory," arXiv:hepth/0309063.

[45] A. B. Zamolodchikov and A. B. Zamolodchikov, "Liouville field theory on a pseudosphere," arXiv:hep-th/0101152.

[46] M. L. Mehta, "Random Matrices," Academic Press, 1991.

[47] E. Brezin, C. Itzykson, G. Parisi and J. B. Zuber, "Planar Diagrams," Commun. Math. Phys. 59, 35 (1978).

[48] A. P. Polychronakos, arXiv:hep-th/9902157.

[49] A. Jevicki and B. Sakita, "The Quantum Collective Field Method And Its Application To The Planar Limit," Nucl. Phys. B 165, 511 (1980).

[50] I. Andric, A. Jevicki and H. Levine, "On The Large N Limit In Symplectic Matrix Models," Nucl. Phys. B 215, 307 (1983).

[51] G. W. Moore and N. Seiberg, "From loops to fields in 2-D quantum gravity," Int. J. Mod. Phys. A 7, 2601 (1992).

[52] A. P. Polychronakos, "Exchange Operator Formalism For Integrable Systems Of Particles," Phys. Rev. Lett. 69, 703 (1992) arXiv:hep-th/9202057.

[53] L. Brink, T. H. Hansson and M. A. Vasiliev, "Explicit solution to the N body Calogero problem," Phys. Lett. B 286, 109 (1992) [arXiv:hep-th/9206049].

[54] G. W. Moore, "Double scaled field theory at c $=1$," Nucl. Phys. B 368, 557 (1992).

[55] F. D. Haldane, "'Fractional Statistics' In Arbitrary Dimensions: A Generalization Of The Pauli Principle," Phys. Rev. Lett. 67, 937 (1991).

[56] Y. S. Wu, "Statistical distribution for generalized ideal gas of fractional statistics particles," Phys. Rev. Lett. 73, 922 (1994).

[57] D. J. Gross and I. R. Klebanov, "One-Dimensional String Theory On A Circle," Nucl. Phys. B 344, 475 (1990).

[58] O. Bergman and M. R. Gaberdiel, "Dualities of type 0 strings," JHEP 9907, 022 (1999) arXiv:hep-th/9906055.

[59] E. Dudas, J. Mourad and A. Sagnotti, "Charged and uncharged D-branes in various string theories," Nucl. Phys. B 620, 109 (2002) arXiv:hep-th/0107081.

[60] C. Ahn, C. Rim and M. Stanishkov, "Exact one-point function of N = 1 super-Liouville theory with boundary," Nucl. Phys. B 636, 497 (2002) arXiv:hep-th/0202043]. 
[61] T. Fukuda and K. Hosomichi, "Super Liouville theory with boundary," Nucl. Phys. B 635, 215 (2002) arXiv:hep-th/0202032.

[62] R. C. Myers and V. Periwal, "Exactly Solvable Oriented Selfdual Strings," Phys. Rev. Lett. 64, 3111 (1990).

[63] T. R. Morris, "Checkered Surfaces And Complex Matrices," Nucl. Phys. B 356, 703 (1991).

[64] A. Anderson, R. C. Myers and V. Periwal, "Complex Random Surfaces," Phys. Lett. B 254, 89 (1991).

[65] T. Yamamoto, "Multicomponent Calogero model of B(N) type confined in harmonic potential," arXiv:cond-mat/9508012.

[66] P. K. Ghosh, A. Khare and M. Sivakumar, "Supersymmetry, Shape Invariance and Solvability of $A_{N-1}$ and $B C_{N}$ Calogero-Sutherland Model," Phys. Rev. A 58, 821 (1998) arXiv:cond-mat/9710206. 